\title{
Classification Precision Analysis on Different Fusion Algorithm for ETM + Remote Sensing Image
}

\author{
Huoqian Luo ${ }^{1, a}$ \\ 1 Workstation of Academician and Experts, Fujian College of Water Conservancy and Electric \\ Power, Yong' an, 366000 \\ ${ }^{a}$ email
}

Key words: Fusion algorithm, ETM +, Remote sensing image fusion, The classification accuracy

\begin{abstract}
With ETM+ data (Nantai Island Fuzhou), several fusion algorithms (PCA, MLT, Brovey Transform, HIS) have been applied in image fusion. After image fusion, some criterions (Spectral fidelity, High spatial frequency information gain, and Classification accuracy, Etc.) have been utilized to evaluate the effect of fusion. Based on the fusion image, unsupervised classification and sequential accuracy assessment have been applied to it. According to the experiment result mentioned above, the paper provides a methodology reference of image fusion to user.
\end{abstract}

\section{Introduction}

Data Fusion is one of the hotspots of remote sensing research. In recent years, domestic and foreign scholars have made a number of remote sensing classical fusion algorithms, such as: a variety of ratios and weighted multiplication, Brovey transformation, high-pass filtering, HIS transformation, principal component analysis and wavelet transform fusion method, and these methods improved algorithms. In this study, March 4, 2001 in Fuzhou Nantai Island ETM + remote sensing images based on data, using principal component analysis (PCA), the product of method (MLT), Brovey HIS transform and transform different spatial resolution based on the same band sensors ( That panchromatic and multispectral bands) fusion experiment, and unsupervised image classification and evaluation of the classification accuracy fused to analyze the effects of different fusion algorithms ETM + remote sensing image classification accuracy.

\section{Main Fusion Algorithm}

Principal component analysis (PCA) is a multi-dimensional (multi-band) on the basis of the statistical characteristics of orthogonal linear transformation, mathematically known as K-L transform [1]. After PCA image fusion includes two of the original image with high spatial resolution and high spectral resolution characteristics, retained the original image of the high-frequency information, detailed features of its objectives clearer, more abundant spectral information [2]. Specific steps using principal component analysis fusion method [3] as follows:

1) Calculation participation n-band multi-spectral image fusion of the correlation matrix;

2) calculated by the correlation matrix eigenvalues $\lambda i$ and eigenvectors $\zeta_{i}(i=1, \ldots, n)$;

3) The characteristic values according to descending order of that $\lambda 1>\lambda 2>\ldots>\lambda$, eigenvectors $\zeta$ i also make corresponding changes;

4) calculated principal component video:

$$
\mathrm{PCk}=\Sigma \mathrm{i}=1 \text { ndi } \zeta \mathrm{ik}(1)
$$

Where: $\mathrm{k}$ main components of ordinal numbers $(\mathrm{k}=1, \ldots, \mathrm{n})$; PCk main component; $\mathrm{i}$ is input bands; $\mathrm{n}$ is the total number of bands in multispectral images; di data value $\mathrm{i}$ band multispectral


component, the first principal component variance of the maximum image which contains a lot of information (mainly the spatial information), while the spectral information is the main image stored in the other components .

5) The spatial registration of the panchromatic image with the first principal component as 
histogram matching;

6) panchromatic image histogram matching generated instead of the first principal component, and compare it with the rest of the main components of the inverse transform principal component analysis, to obtain high-resolution multi-spectral image fusion.

MLT (Multiplication) algorithm is a high resolution image pixels on each band respectively on the low resolution image corresponding to the pixel multiplied, thereby improving the spatial resolution of low-resolution images. Its expression is:

$$
B_{M L T i}=\sqrt{a \times B_{\text {lowi }} \times b \times B_{\text {high }}}
$$

Where: BMLTi is through the $\mathrm{i}$-th band MLT method after fusion, $\mathrm{B}_{\text {lowi }}$ resolution image for the $\mathrm{i}_{-}$th band of low spatial, $\mathrm{B}_{\text {high }}$ high-resolution image space. Since the product of the gray value image is too large, and therefore take the product of the square root. a and b are weighting coefficients, practical applications

May be needed for different bands normalized weighting of $a$, b takes different weights, but in order to make the results representative, using affirmative treatment in this study.

Brovey transform fusion method is relatively simple fusion method, it is displayed as an image RGB color multi-spectral bands normalized, high resolution panchromatic and multispectral images of red, green, and blue band of the respective proportion multiplied complete fusion [4]. Holding high-resolution image space texture, better able to edge out the difference between different land types. The formula is:

$$
B_{i_{-} \text {new }}=\frac{B_{i_{-} m}}{\left(B_{r_{-} m}+B_{g_{-} m}+B_{b_{-} m}\right)} \times B_{\text {high }}
$$

Where: band value $B_{i_{-} \text {new }}$ Representative fused $(i=1,2,3) ; B_{r_{-} m}, B_{g \_m}, B_{b_{-} m}$ represent low-resolution multi-spectral images of arbitrary, $\mathrm{B}_{\text {high }}$ represents the high resolution panchromatic image.

In image processing, often applied color coordinate system (or color space) there are two kinds: the three primary colors (RGB) space; HIS color system (Munsell color system). In general, the image of the RGB color space is not fusion, and HIS systems on the contrary, it clearly illustrates the characteristics of the particular color channel. In color science, usually called the space to transform HIS HIS space conversion from RGB, the formula is:

$$
\left\{\begin{array}{l}
{\left[\begin{array}{l}
I \\
v_{1} \\
v_{2}
\end{array}\right]=\left[\begin{array}{l}
\frac{1}{\sqrt{3}} \frac{1}{\sqrt{3}} \frac{1}{\sqrt{3}} \\
\frac{1}{\sqrt{6}} \frac{1}{\sqrt{6}}-\frac{2}{\sqrt{6}} \\
\frac{1}{\sqrt{2}}-\frac{1}{\sqrt{2}} 0
\end{array}\right] \cdot\left[\begin{array}{l}
R \\
G \\
B
\end{array}\right]} \\
S=\sqrt{v_{1}^{2}+v_{2}^{2}} \\
H=\arctan \left[\frac{v_{1}}{v_{2}}\right]
\end{array}\right.
$$

Where: I represents brightness of the color, all the energy and spatial information mainly reflects the image reflected image feature included, corresponding to the image of the ground resolution; $\mathrm{H}$ represents chroma, refers to the composition of the primary color wavelength, red, green blue proportion determined; $\mathrm{S}$ represents the saturation, the purity of the representative color, $\mathrm{H}$ and $\mathrm{S}$ represents the spectral resolution of the image [6].

That is the reverse transformation from the HIS space to RGB color space conversion, called RGB conversion, the formula is:

$$
\left[\begin{array}{l}
R \\
G \\
B
\end{array}\right]=\left[\begin{array}{l}
\frac{1}{\sqrt{3}} \frac{1}{\sqrt{6}} \frac{1}{\sqrt{2}} \\
\frac{1}{\sqrt{3}} \frac{1}{\sqrt{6}}-\frac{1}{\sqrt{2}} \\
\frac{1}{\sqrt{3}}-\frac{2}{\sqrt{6}} 0
\end{array}\right] \cdot\left[\begin{array}{l}
I \\
V_{1} \\
V_{2}
\end{array}\right]
$$

Fusion use HIS transformation, the first multi-spectral image use HIS conversion from RGB to HIS space transform the system, while the single-band high-resolution image through gray stretch, 
so that the mean and variance of gray and HIS space the luminance component of the image similar to the image to increase 2 between a correlation and suppress flare effect; then stretched panchromatic image as a new generation of the luminance component into the HIS, are transformed restored to the original space .

\section{Fusion Experimental Results Analysis and Evaluation}

In this paper, Fuzhou has over 2001 registration ETM + images were fused by the above method, the results shown in Figure 1.


Figure 1 Original image after image fusion false color composite image (RGB543)

(A) Original image Figure (b) PCA fusion image maps (c) MLT fusion image maps (d) Brovey fusion image maps (e) HIS fusion image map

Evaluate the effects of fusion, are generally subjective evaluation and objective evaluation. Subjective evaluation is analyzed by visual effects; objective evaluation using image maps for analysis and evaluation, usually from the spectral fidelity, root mean square deviation aspects (Root Mean Square Error, RMSE), the degree of integration of high frequency information, etc. comprehensive quantitative analysis and evaluation.

The contents of subjective evaluation of the following: whether the fusion image registration; fused images overall color is the same with natural color; Analyzing Fusion overall image brightness, contrast, if appropriate; integration of image texture and color information is rich, spatial information is lost; fusion whether the reduced image sharpness, edge feature image is clear [7]. From the visual effects in Figure 1 to see the sheets of image maps, a variety of integration methods to improve the visual effect of the original panchromatic image and multi-band composite image as a whole. As shown in Table 1 below:

Table 1 different fusion algorithm results in Fig subjective evaluation

Fusion Image fusion algorithm used Comparative Analysis of the original image map

Color, hue similar to principal component analysis described fusion can better maintain the spectral characteristics of the original image; (b FIG) in different colors to

Principal Component Analysis distinguish distinct categories, convergence at the clear lines, the feature will not form a clear image of jagged edges, improved spatial resolution of surface features, detailed features clearer, more abundant spectral information. 
With the original (a map) comparison can clearly see that the more significant changes in the color of the graph, color tone, there is no

Product transformation

Brovey transformation

HIS conversion method better maintained spectral characteristics of the original image; color differently class distinction and connection with the original boundaries (a map) relatively more clear, but the effect is not as good as (b FIG), spectral information and clarity is relatively weak.

Color, tint, with the original (a map) closest to illustrate the Brovey best method to maintain the spectral characteristics of the original image; (d FIG) in different colors to distinguish classes obvious convergence at the boundaries clear, rich feature of the spectral information; the spatial resolution (c FIG) have significantly higher than the original image (a map).

Color, tint, and (c FIG) were similar, not fully maintain the spectral characteristics of the original image, but its brightness and contrast ratio (c Figure) high, you can better maintain the multi-spectral characteristics; spatial resolution than the original image (a FIG) has improved greatly, and compared with other imaging FIG fused around the class convergence at No jagged, improve the spatial resolution of the best.

Each band spectral fidelity fusion image by calculating the mean deviation of their brightness between the original image (mean difference) is measured (Table 2). Painted line chart (Figure 2) can visually see their trends, and the root mean square error (RMSE) can measure the similarity of n-dimensional space of two measures vectors [5], the formula is:

$$
R M S E=\sqrt{\sum_{i=1}^{n} \frac{\left(B_{\text {mean }_{i}}^{\prime}-B_{\text {mean }}\right)^{2}}{n}}
$$

Where $\mathrm{n}$ is the number of bands for the first $B_{\text {mean }}^{\prime}$ mean image fusion band, $B_{\text {mean }}$ the original image of the i-band average. RMSE smaller the value, the degree of deviation from the original spectral image fusion image smaller, higher fidelity.

Table 2 Mean brightness of the original image and the fused image of each band

\begin{tabular}{cccccccccccccc}
\hline & Wave & Mean & Wave & Mean & Wave & Mean & Wave & Mean & Wave & Mean & Wave & Mean & RMS \\
\hline Origin & 84.1 & & 68.18 & & 66.06 & & 46.17 & & 62.50 & & 47.33 & & \\
PCA & 95.33 & -11.23 & 83.12 & -14.9 & 71.15 & -5.09 & 84.15 & -37.9 & 82.83 & -20.3 & 75.38 & -28.0 & 22.43 \\
MLT & 32.67 & 51.43 & 29.85 & 38.33 & 25.44 & 40.62 & 34.33 & 11.84 & 20.37 & 42.13 & 15.65 & 31.68 & 38.04 \\
Brove & & & & & 47.91 & 18.15 & 52.63 & -6.46 & 38.45 & 24.05 & & & 17.79 \\
HIS & & & & & 69.79 & -3.73 & 89.78 & -43.6 & 59.29 & 3.21 & & & 25.34 \\
\hline
\end{tabular}




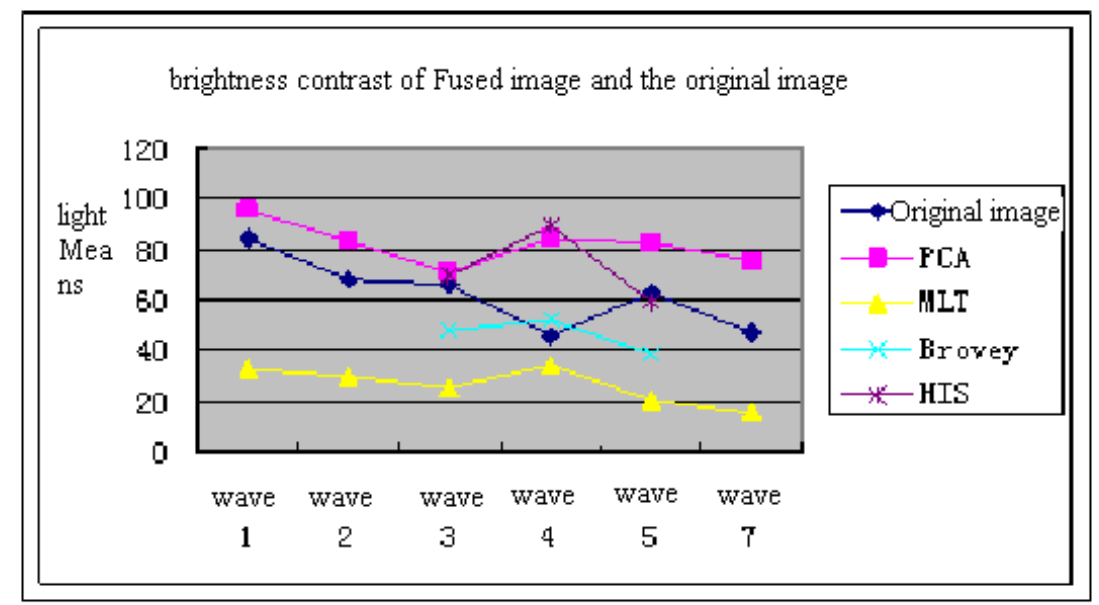

Figure 2 Mean luminance contrast original image and fusion image

Table 2 and Figure 2 show: Means and closer to the original image Brovey law, RMSE minimum value, indicating Brovey fusion of the spectrum of the original image caused by distortion of the minimum; RMSE value of the product of the largest law shows its spectral distortion caused by the largest; the first three bands PCA fusion method and the mean is closer to the original image, RMSE values centered, indicating that PCA fusion method can better retain the spectral information; information on spectral distortion is less than the product of HIS fusion method, but greater than PCA method .

Integration of high-resolution images into the texture information through the integration of the correlation coefficient between the image and the high resolution image to determine [8], and with the average correlation coefficient and RMSE to measure. The average value of the correlation coefficient, the smaller the RMSE deviation, the more into the high-resolution information.

Table 3 bands fused image and the original image with the Pan-band correlation coefficient

\begin{tabular}{ccccccccc}
\hline wave & wave1 & wave2 & wave3 & wave4 & wave5 & wave7 & Means & RMSE \\
\hline Origin & 0.384 & 0.528 & 0.619 & 0.68 & 0.736 & 0.716 & 0.6105 & 0.3895 \\
PCA & 0.406 & 0.592 & 0.693 & 0.693 & 0.78 & 0.786 & 0.65833 & 0.3417 \\
MLT & 0.86 & 0.857 & 0.837 & 0.892 & 0.865 & 0.827 & 0.85633 & 0.1437 \\
Brovey & & & 0.889 & 0.773 & 0.868 & & 0.84333 & 0.1567 \\
HIS & & & 0.914 & 0.935 & 0.804 & & 0.88433 & 0.1157 \\
\hline
\end{tabular}

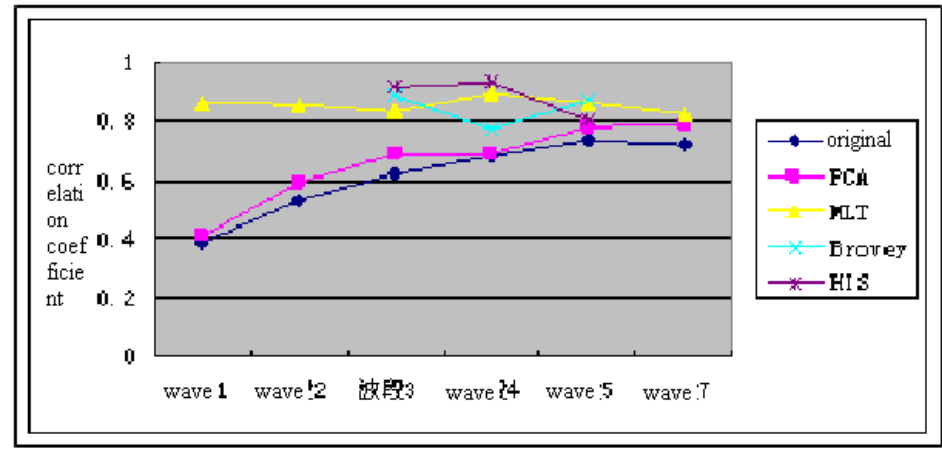

Table 3 bands fused image and the original image with the Pan-band correlation coefficient

Table 3 and Figure 3 shows: MLT fusion image, Brovey HIS fusion image fusion and image correlation is relatively high, indicating that the fusion of these three images are effectively integrated into the high-resolution image information. Among them, the highest correlation HIS image, RMSE only 0.1157 , followed by the MLT, RMSE is 0.1437 .

\section{Image Classification}


In this paper, respectively, using the above method of generating different fusion fusion image maps unsupervised classification results shown in Figure 4.
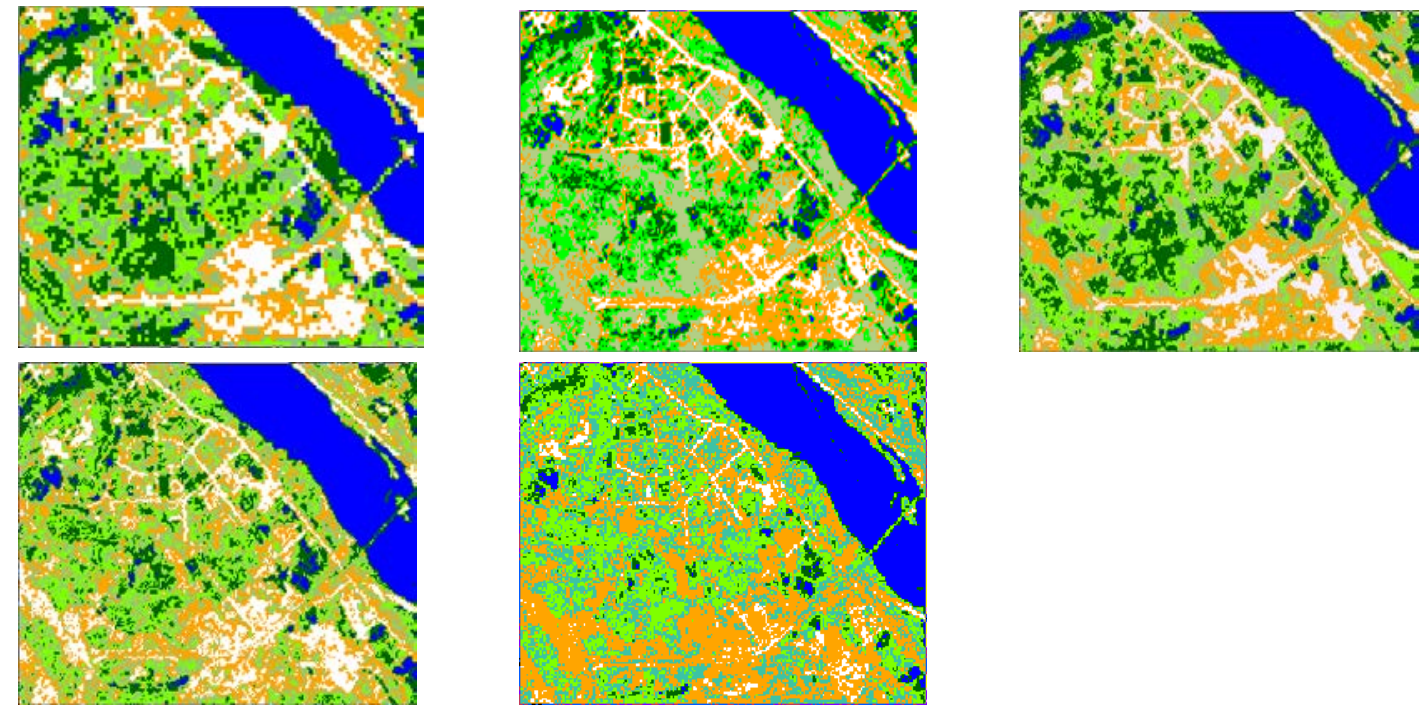

Fig. 4 image classification map of the study area (range interception section)

(A) the original image classification map (b) PCA fusion image classification map (c) MLT fusion image classification map (d) Brovey fusion image classification map (e) HIS fusion image classification map

Preliminary visual discrimination can be seen free fusion image classification results better than the original image. Since not into the high-resolution images, the original image classification results showed polygons rough edges, serrated obvious, built-up areas difficult to identify streets, rivers, jagged edges. The fusion image misclassification phenomenon reduction, streets clear, polygon edge smoothing, classification accuracy and spatial resolution are significantly improved. Comparison of FIG. 4 (b), (c), (d), (e), apparent PCA, Brovey fusion method of classification good classification results than the product of the fusion method.

To further validate the accuracy of classification, to establish the accuracy of estimation error matrix. Error matrix generated using pixel sampling when sampling is required to determine the sampling points and the sampling method, to determine the pixel-by-point reference (actual) category. Experimental support in ERDAS Imagine 8.7, the use classification module (Classifier) in the Accuracy Assessment function random sampling point by point and then determine the reference category. Reference category is determined using land-use maps and visual interpretation combined, point by point to accurately determine the sampling points, and then do the evaluation were obtained to assess the accuracy of the preliminary classification error matrix diagram, and calculates the error matrix based on the overall accuracy and Kappa coefficient [9], as shown in table 4 and Figure 5.

Kappa analysis is a multivariate statistical method to evaluate the classification accuracy, Kappa coefficient represents assessment and classification error than to reduce the proportion of completely random assortment production. The total difference between precision and accuracy Kappa coefficient is used only a total number of cells located on the diagonal, and the Kappa coefficient takes into account both the diagonal correctly classified pixels, but not on the diagonal into account a variety of drain points and misclassification error.

Table 4 Evaluation of Accuracy of different fusion methods

\begin{tabular}{ccc}
\hline & $\begin{array}{c}\text { Overall } \\
\text { accuracy }\end{array}$ & $\begin{array}{c}\text { KAPPA } \\
\text { Coefficient }\end{array}$ \\
\hline Original image & 0.6167 & 0.5328 \\
PCA & 0.6571 & 0.59 \\
MLT & 0.6333 & 0.5609 \\
Brovey & 0.7 & 0.6373 \\
HIS & 0.645 & 0.5881 \\
\hline
\end{tabular}




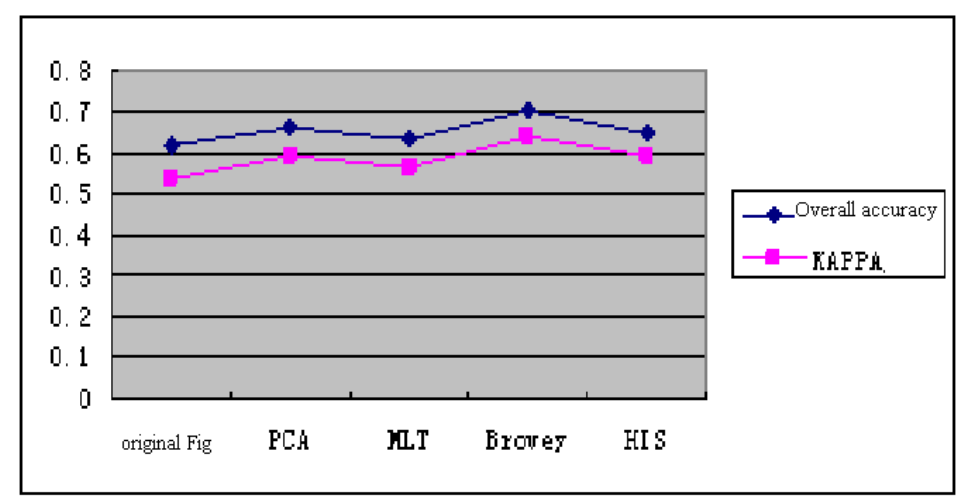

Fig.5 different fusion classification accuracy comparison chart

Classification results show that the classification accuracy of the image classification accuracy higher than the original image are after integration. Wherein Brovey fusion highest classification accuracy reached $70 \%$, compared with the original image improved 8.33 percentage; Kappa coefficient is $0.6373,0.1045$ improved than the original image. Followed by the PCA fusion method, accuracy of $65.71 \%$, kappa coefficient is 0.59 ; HIS fusion method of classification accuracy in general; the four fusion method MLT fusion classification accuracy weakest.

\section{Conclusion}

Fusion use ETM + images for automatic classification can significantly improve the classification accuracy, because the use of homologous integration of image sensors to be classified, to avoid the image sensor parameters heterologous fusion exists, and registration phase differences may lead to a series of classification reduced accuracy factors. Fusion image classification accuracy is not only affected by the spectral fidelity, but also affected to some extent the high frequency spatial information into the degree. Therefore, in selecting Fusion Algorithm for classification, and comprehensive consideration of these two factors. Multi-spectral information fusion and PCA fusion method Brovey more reserved, but HIS fusion method, MLT fusion method and Brovey integration into the law of large high-resolution information. Therefore, considering the spectral fidelity, high-frequency and spatial information into the classification accuracy of these factors, it can initially considered: Brovey fusion method for ETM + image fusion can better achieve enhanced visual effects and improve the classification accuracy to identify, PCA fusion method for improving the accuracy of the classification of the contribution ranked second, while the classification results HIS fusion method and MLT fusion method is relatively weak.

\section{Acknowledgements}

In the research process of this study, Teacher Chen Wenhui and Ding Fengand, Student Xie Aiying and Qi Yu have gived continuously support. I express my gratitude for the two teachers and two students.

Minjiang Shaxi digital watershed model and its application (Water and Sediment Sciences, Tsinghua University and the Open Research Laboratory Hydropower Project, No: sklhse-2014-B-06);

Burned temporal remote sensing characteristic spectrum of forest restoration ( ID: XY1502B);

\section{References}

[1] Zhou Shijian, Zang Deyan, Lu Tieding. Survey adjustment models in a variety of models equivalent transformation of the relationship between [J]. College of Surveying and Mapping, 2001,18 (1): 123.

[2] Zhou Wanpeng, Zhu Qiangmin, Wu Rengui. ETM + Image Data Fusion cover classification [J]. Journal, 2004, 27 (4): 333-340. 
[3] Fan Xuyan, Fu Chunlong, Dan Jihai. Remote sensing image simulation based on principal component analysis, true-color fusion method [J]. Surveying and Mapping Science \& Technology, 2006,23 (4): 287-289,292.

[4] Fan Xuyan, Yin Lianwang, Fu Chunlong. QuickBird Remote Sensing Image Data Fusion Method [J] Equipment Command \& Technology, 2006, 17 (3): 81-85.

[5] Xu Hanqiu. Landsat Landsat image fusion and classification study ETM + Auto [J]. Remote Sensing, 2005,9 (2): 186-194.

[6] Zhou Qianxiang, Jing Zhongliang, Jiang Shizhong. theoretical resolution remote sensing image fusion method of different spectral space [J]. Remote Sensing Technology and Application, 2003, 18 (1): 41-45.

[7] Liu Xiaolong. Multi-source remote sensing image information holding type Fusion Technology [J]. Chinese Journal of Image and Graphics, 2001,6 (7): 636-641

[8] Bretschneider T, Kao O. Image Fusion in Remote Sensing [A]. Proceedings of the 1st Online Symposium of Electronic Engineers.2000,1-8.

[9] Danf Anrong, Wang Xiaodong, Chen Xiaofeng. Erdas Imagine remote sensing image processing method [M]. Beijing: Tsinghua University Press, 2003. 